\title{
Modeling the interfaces in masonry structures
}

\author{
Frédéric Lebon* \\ Aix-Marseille University, LMA, UPR7051, CNRS, Centrale Marseille, 13402 \\ Marseille, France \\ frederic.lebon@univ-amu.fr
}

\begin{abstract}
This chapter deals with some models for interfaces in the case of masonry structures. Some experimental studies are recalled in the first part. In the second part, four interface models are presented.
\end{abstract}

\section{Introduction}

The aim of the chapter is to present some ideas for modeling interfaces in masonry structures. Devising means of modeling interfaces between solids in structural assemblies is obviously now of great importance in the fields of mechanical technology and civil engineering. These interfaces contribute crucially to the strength of many structures, such as optics lenses, airplanes, asphalt pavements and masonry, for example. It is therefore necessary to develop rather fine models. One of the main problems which often arises in this context is that of developing a unified theory: from the tribological point of view, the contact is often unique, in the sense that it depends on the materials, roughness, wear, etc., and especially on the mechanical system involved. The problem of cracking in pavements is obviously quite different from that of the cornering of an airplane tire. Another problem is due to the smallness of the interface in comparison with the size of the structure, as well as the possibly weak mechanical characteristics (in the case of old mortar, for example). A large number of studies have been devoted to the behavior of interfaces. Two main modeling approaches used for this purpose are phenomenological modeling and deductive modeling. In the first approach, the thickness of the interface is taken to be zero and the mechanical properties are obtained from physical considerations and experiments (see for example Frémond (1987); Point and Sacco (1996); Freddi and Frémond (2006); Raous (2011); Bonetti and Frémond (2011) and references therein). The

\footnotetext{
${ }^{*}$ The author thanks F. Fouchal, C. Pelissou, A. Rekik, R. Rizzoni and I. Titeux for their contribution in this work
} 
second approach consists in focusing on the thin layers of material at the micro-mechanical level, which are usually called the interphase. The mechanical parameters of the interface model, characterized by zero thickness, are identified on the basis of the parameters of the material constituting this interphase. These boundary conditions have been extensively used to model imperfect interface properties. One of the method commonly used to model interface conditions is based on the use of asymptotic techniques (see for example Klarbring (1991); Licht and Michaille (1997); Lebon et al. (1997, 2004); Lebon and Ronel (2007); Lebon and Rizzoni (2008) and references therein), in order to include microscopic considerations in the interface model.
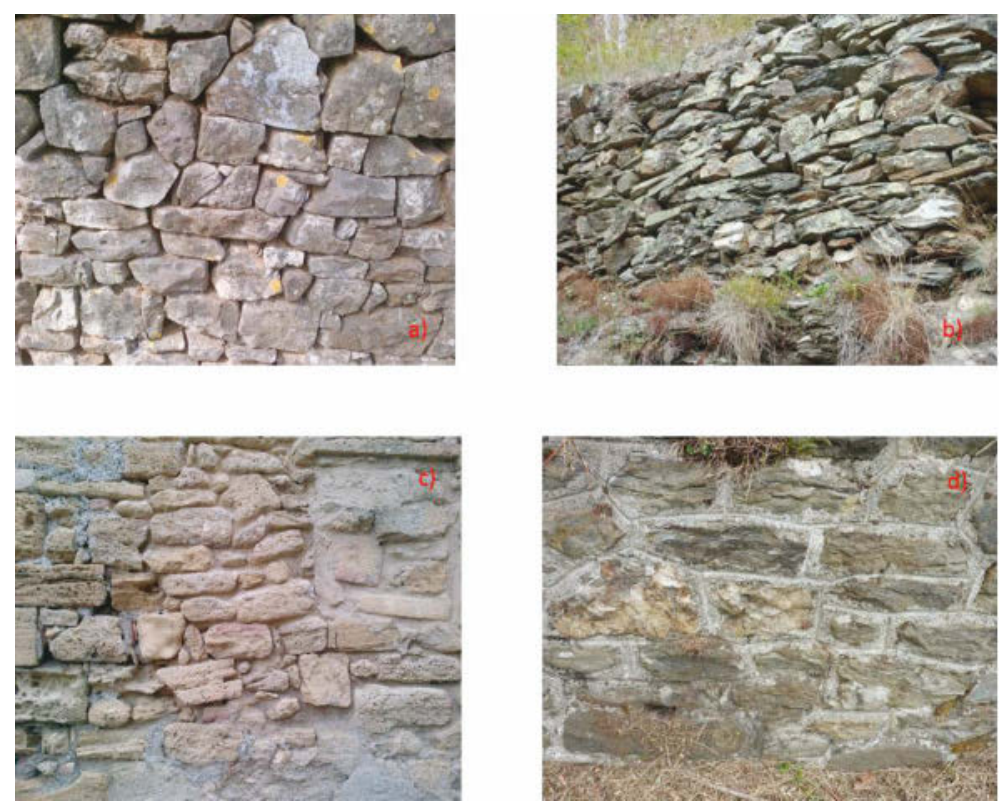

Figure 1. Irregular type of masonry a) b) d) Typical Lozerian structures, France c) Miramas-Le-Vieux, France.

Obviously, one of the problem which arises when modeling interfaces in masonry is due to the the irregularity of the structures (see figure 1). In this chapter, we will deal only with fairly regular structures (see figure 2). Another problem arises with the constitutive equation for the blocks because stones are quite rigid blocks (see Lucchesi et al. (2008); Como (2010)). In 

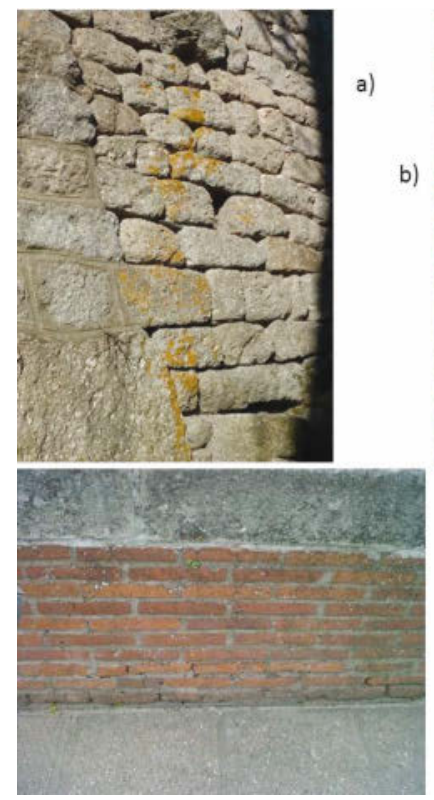

b)

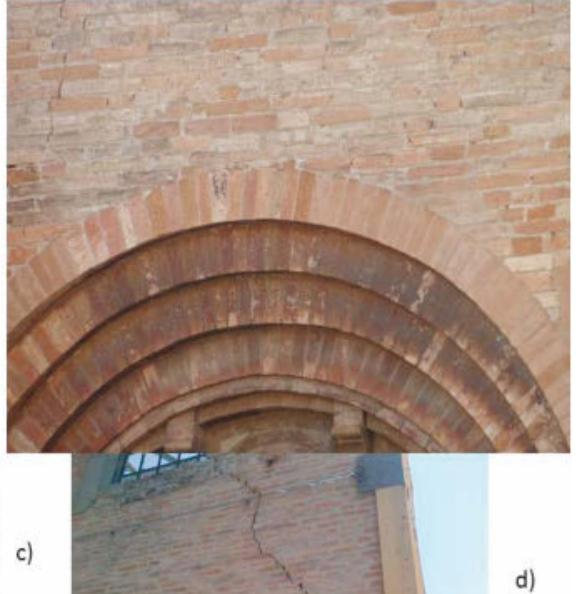

d)

Figure 2. Examples of regular masonry a)Lozere, France b) Bologne, Italy c) Albi, France d) Ferrara, Italy.

what follows, we will deal only with deformable elastic bricks.

The last main problem depends on whether or not mortar is present in the structure and if so, how to write the constitutive equation for the mortar. If there is no mortar, the contact between blocks can be assimilated to dry friction. In the presence of mortar, it is necessary to model this component. The thickness of the mortar is also an important modeling parameter. If the mortar is thin, an interface law can be used directly for this purpose (see figure 3). If the thickness of the mortar is not negligible, it is necessary to introduce some additional considerations in order to account for the interactions between the bricks and the mortar (see figure 4).

In this chapter, it is proposed to present some interface laws. In the first part, the classical phenomenological law of unilateral contact with dry friction is recalled. In the second part, a phenomenological law of adhesion is presented and modeled based on the adhesion variable introduced by Fremond (Frémond (1987)). Two deductive interface laws are presented: the first one is linear and the second one is non linear and takes the damage which occurs at interface of this kind into account. The last modeling 


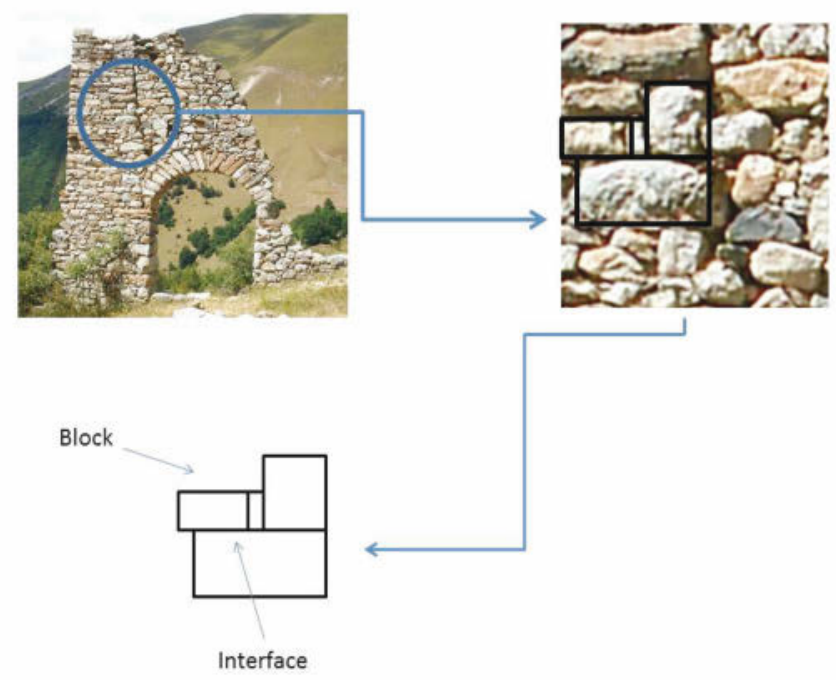

Figure 3. A wall without mortar: a study at local level.

approach presented here includes mechanical processes responsible for the failure, such as the unilateral contact, the friction at the microscopic scale and the normal and tangential damage. The four models discussed in this chapter were developed in the context of multibody mechanics.

This paper is composed of three parts. In the first part, experimental results are recalled. The second part is devoted to the modeling of friction between blocks with no mortar. In the third part of the paper, the phenomenological model of adhesion is presented in the form of two imperfect interface models based on the use of asymptotic techniques. Some numerical examples are provided.

\section{Some comments on experimental results}

\subsection{Brick-brick interactions: dry friction}

Michel Jean (Jean and Moreau (1994)) conducted experiments on a small wall (figure 5) consisting of 105 rigid blocks. each of which was $49 \mathrm{~mm}$ high, and $124 \mathrm{~mm}$ wide ( $62 \mathrm{~mm}$ in the case of half bricks). The wall was set on 


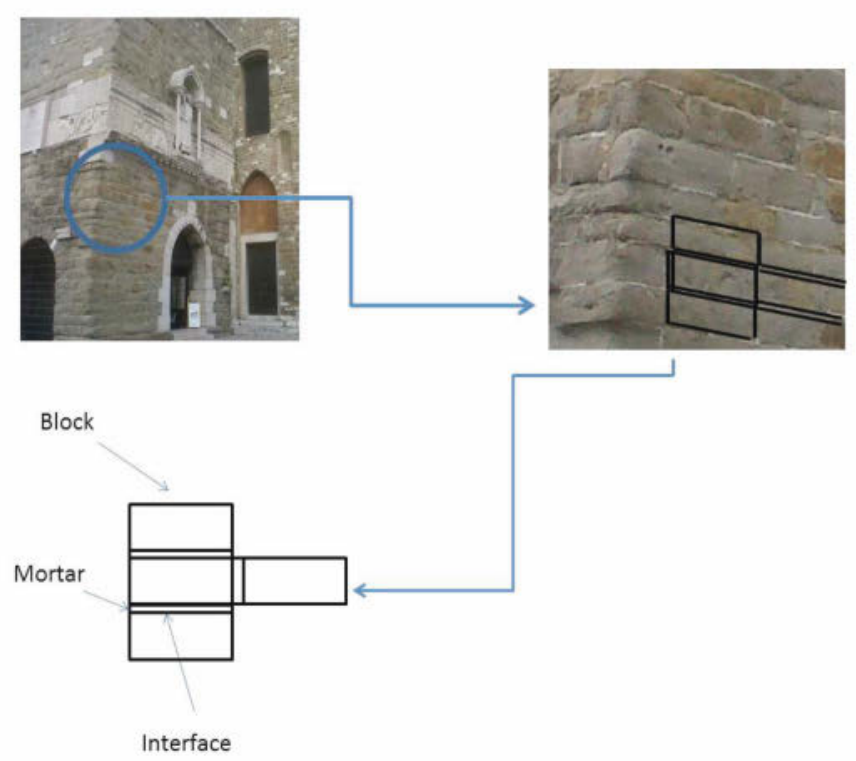

Figure 4. A wall with mortar: a study at local level.

a rigid foundation. The left half of the rigid foundation could be moved down. The total possible displacement was $6 \mathrm{~cm}$.

Figure 5 shows the displacement of the blocks. Note that the interactions between the blocks are limited to contact (there is no penetration) and dry friction. The displacement of the blocks is strongly heterogeneous, especially along the diagonal.

\subsection{Brick-mortar interactions}

This section deals with the behavior of deformable blocks. The local behavior of the interfaces between full and hollow bricks and mortar joints, which are typical quasi-brittle interfaces (see Gabor (2002); Gabor et al. (2006)), has been studied by various authors. We will attempt here to summarize the results obtained.

The experimental device developed by Fouchal (2006); Fouchal et al. (2009) (figures. 6 and 7) was designed to study on the local scale the shear behavior of a simple assembly consisting of two and three full or hollow bricks $(210 \times$ $50 \mathrm{~mm}$ ) connected by a mortar joint $10 \mathrm{~mm}$ thick. The samples were subjected to a monotonous increasing load up to failure. 


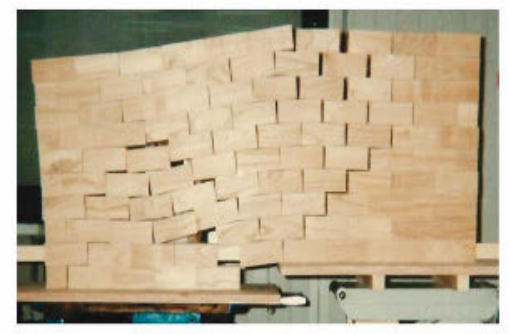

Figure 5. Experimental device involving 105 blocks (Jean and Moreau (1994))
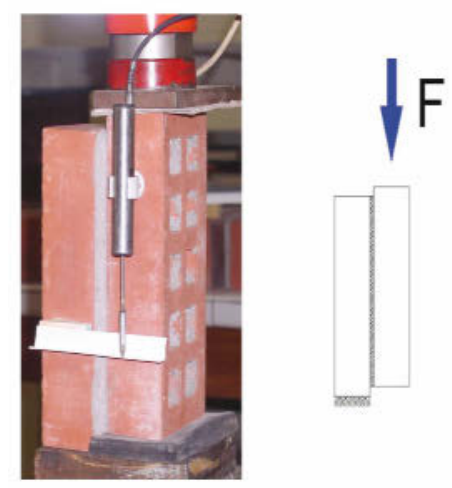

Figure 6. Experimental device involving two bricks

The following findings were obtained (see also figure 8):

- rigid elastic behavior up to the failure, followed by friction sliding behavior;

- the behavior of full bricks was fragile beyond the limit strength;

- the behavior of hollow bricks was quasi-fragile beyond the limit strength;

- hollow brick samples showed great dispersion, mainly due to the non uniform distribution of the mortar spikes and local defects in the com- 

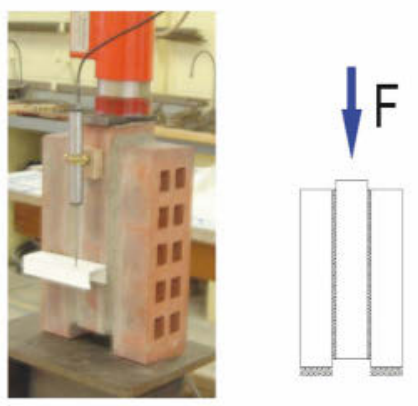

Figure 7. Experimental device involving three bricks

ponents of the bricks;

- samples consisting of two and three bricks showed similar behavior. The choice of basic cell therefore has no effect on the local scale.

\section{Masonry structures without mortar: dry friction modeling (model 1)}

This section focuses on structures devoid of mortar, or including mortar with a very low stiffness. In a first approach, the contact is assumed to involve dry friction with no penetration between the blocks.

For the sake of simplicity, we have adopted the framework of contact between two deformable solids (see figure 9). The contact can be defined by a punctual correspondence between two surfaces in contact $\Gamma_{c}^{1}$ and $\Gamma_{c}^{2}$ belonging to the domains $\Omega^{1}$ and $\Omega^{2}$ of $\Re^{d}(d=2,3)$, respectively. We assume that initially $\Gamma_{c}=\Gamma_{c}^{1}=\Gamma_{c}^{2}$. The relative displacement between two points located on the two surfaces in contact is denoted by $[u]$ with $[u]=u^{1}-u^{2}$. Let $F$ be the density of the contact forces. We take $n^{1}$ and $n^{2}$ to denote the external unit normal vectors to the boundaries of the two domains. The decomposition into normal and tangential parts is written:

$$
\begin{gathered}
{[u]=\left[u_{N}\right] n^{1}+\left[u_{T}\right] \quad \text { with } \quad\left[u_{N}\right]=[u] . n^{1}} \\
F=F_{N} n^{1}+F_{T} \quad \text { with } \quad F_{N}=F . n^{1}
\end{gathered}
$$




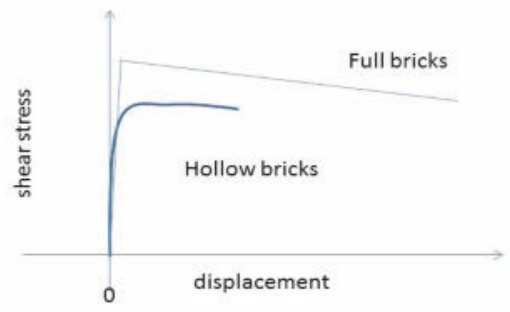

Figure 8. Typical behavior of the mortar/brick interface (with full and hollow bricks)

\subsection{Unilateral contact}

The contact conditions (Signorini conditions) are written:

$$
F_{N} \geq 0 ; \quad\left[u_{N}\right] \geq 0 ; \quad F_{N}\left[u_{N}\right]=0
$$

\subsection{Dry Friction}

The friction conditions (Coulomb's law) are written:

$$
\begin{aligned}
& \left\|F_{T}\right\| \leq \mu\left|F_{N}\right| \\
& \left\|F_{T}\right\|<\mu\left|F_{N}\right| \Rightarrow \quad \Rightarrow \quad\left[u_{T}\right]=0 \\
& \left\|F_{T}\right\|=\mu\left|F_{N}\right| \Rightarrow \quad \exists \lambda \geq 0, \quad\left[\dot{u}_{T}\right]=\lambda F_{T}
\end{aligned}
$$

where $\mu$ is the friction coefficient.

\subsection{Formulations}

The bodies are assumed to be elastic and the deformations are assumed to be small. In this case, formulation of three kinds can be used: primal (the unknowns are the displacements), dual (the unknowns are the stresses) and mixed formulations (the unknowns are the displacements and the stresses). 


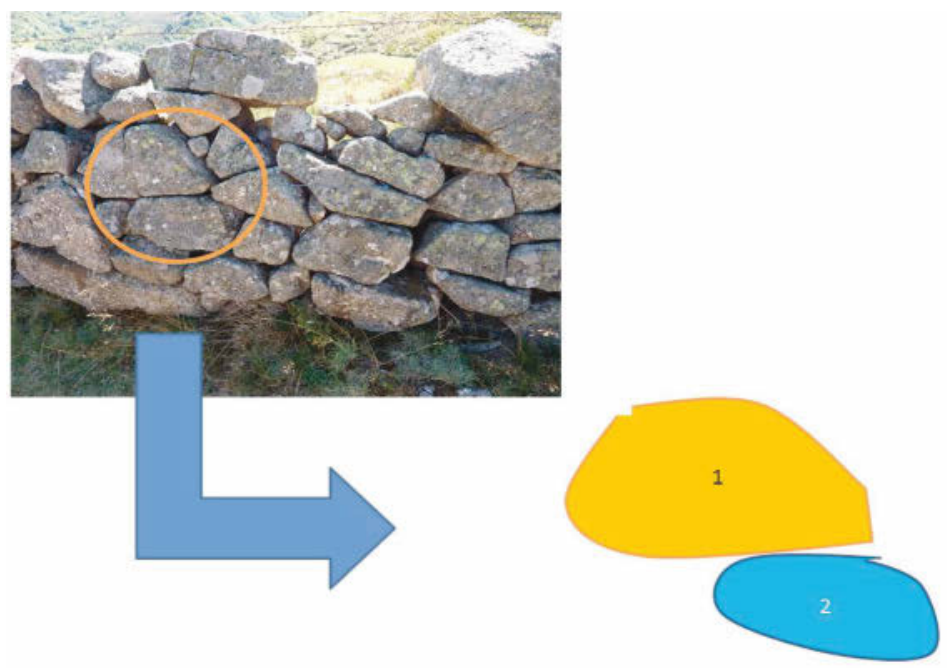

Figure 9. Dry contact between two blocks

In this chapter, two kinds of formulations are presented. Note that there exists a large class of methods for solving problems of this kind (see Raous et al. (1988); Lebon and Raous (1992); Chabrand et al. (1998); Lebon (2003); Fortin et al. (2002); Alart and Curnier (1991); Alart and Lebon (1995); Wriggers (2006) and references therein). Two corresponding algorithms are presented below.

Primal formulation (see Cocu et al. (1995))

Let us take $H^{1}(\Omega)$ to denote the order 1 Sobolev space, and $H$ and $H_{0}$ to denote the subspaces of $\left(H^{1}(\Omega)\right)^{2}$ defined by

$$
\begin{aligned}
H & =\left\{v \in\left(H^{1}(\Omega)\right)^{2}, v=U \text { on } \Gamma_{d}\right\}, \\
H_{0} & =\left\{v \in\left(H^{1}(\Omega)\right)^{2}, v=0 \text { on } \Gamma_{d}\right\},
\end{aligned}
$$

We define $\mathcal{K}$ as the convex of contact of kinematically admissible fields $\mathcal{K}=\left\{v \in H,\left[v_{N}\right] \geq 0\right.$ on $\left.\Gamma_{c}\right\} . u_{0}$ is taken to denote the initial displacements 
fields.

$$
\left\{\begin{array}{l}
u_{0} \in H \text { be given, find } u \in V=H^{1}(0, T ; \mathcal{K}) \text { such that } u(0)=u_{0} \text { in } \Omega \\
a(u, v-\dot{u})+j(u, v)-j(u, \dot{u}) \leq l(v-\dot{u}), \quad \forall v \in H_{0} \\
\int_{\Gamma_{c}} \sigma_{N}(u)\left(\left[z_{N}\right]-\left[u_{N}\right]\right) d l \leq 0, \quad \forall z \in \mathcal{K}
\end{array}\right.
$$

with

$$
\begin{gathered}
j(u, v)=-\int_{\Gamma_{c}} \mu \sigma_{N}(u)\left\|\left[\dot{v}_{T}\right]\right\| d l, \\
l(v)=\int_{\Omega} F v d x+\int_{\Gamma_{f}} f v d l \\
a(u, v)=\int_{\Omega} e(u) A e(v) d x .
\end{gathered}
$$

Using a finite difference, $\dot{u}\left(t^{k+1}\right) \simeq \frac{u^{k+1}-u^{k}}{\Delta t}=\frac{\Delta u^{k}}{\Delta t}$, with $u^{k}=u\left(t_{k}\right)$, at time $t_{k+1}$, we have

$$
\left\{\begin{array}{l}
\text { Find } u^{k+1}=u^{k}+\Delta u^{k} \in \mathcal{K} \text { such that } \\
a\left(u^{k+1}, v-\Delta u^{k}\right)+j\left(u^{k+1}, v\right)-j\left(u^{k+1}, \Delta u^{k}\right) \leq l\left(v-\Delta u^{k}\right), \quad \forall v \in \mathcal{K} .
\end{array}\right.
$$

Note that the results obtained on the existence, unicity and regularity of the solution for this problem have been discussed in Cocu (1984). An example of non uniqueness is given in Hild (2003).

Fixed point Method FPM (see Raous et al. (1988); Lebon and Raous (1992)) In the following sections, the indices $k$ (time) will be omitted (this is a static formulation). The above problem is expressed as a fixed point problem on the sliding limit (Duvaut and Lions (1976); Cocu (1984))

$$
\lambda \longrightarrow-\mu \sigma_{N}(u(\lambda)),
$$

$u$ is the solution of a variational inequation similar to the above one, where $j(.,$.$) is replaced by j($.$) defined by$

$$
j(v)=\int_{\Gamma_{c}} \lambda\left|v_{T}\right| d l
$$

The latter problem is equivalent to the minimization of $L(v)=\frac{1}{2} a(v, v)-$ $l(v)+j(v)$ in $\mathcal{K}$. It is possible to regularize the non-differentiable term 
in the minimization problem, by replacing the absolute value by a smooth function. For example, a suitable value of $\sqrt{x^{2}+\epsilon^{2}}$ or $\epsilon \operatorname{Ln}(\operatorname{ch}(x / \epsilon))$ can be chosen (Lebon (1995)). This regularization procedure does not affect numerical structures. The problem is usually discretized by a linear interpolation (Finite Element Method). Let $h$ be the discretization step. The displacement gives to a vector of dimension $N_{h}$. If $I_{h}$ is the set of degrees of freedom involved in the friction, the fixed point iteration consists in finding the vector of dimension $I_{h}, \lambda_{h}$, which is the fixed point of the previously discretized problem. The algorithm, which is convergent, is written:

0 Initialization $u^{0}$ be given

1 Fixed Point (Iteration k) $\lambda^{k} \longrightarrow-\mu \sigma_{N}\left(u\left(\lambda^{k-1}\right)\right)$

2 Minimization (Relaxation)

A Initialization $u^{k, 0}=u^{k}$

B Resolution (iteration $\mathrm{n},\left(a_{i j}\right)$ is the stiffness matrix)

Case 1 With a normal component of a contact node

$$
\begin{gathered}
u_{i}^{k, n+\frac{1}{2}}=\frac{1}{a_{i i}}\left(f_{i}-\sum_{j=1}^{j=i-1} a_{i j} u_{j}^{k, n}-\sum_{j=i+1}^{j=N_{h}} a_{i j} u_{j}^{k, n+1}\right) \\
\text { If } u_{i}^{k, n+\frac{1}{2}} \geq 0 \text { then } u_{i}^{k, n+1}=0 .
\end{gathered}
$$

Case 2 With a tangential component of a contact node

$$
\begin{array}{r}
u_{i}^{k, n+1}=\frac{1}{a_{i i}}\left(f_{i}-\sum_{j=1}^{j=i-1} a_{i j} u_{j}^{k, n}-\sum_{j=i+1}^{j=N_{h}} a_{i j} u_{j}^{k, n+1}\right. \\
\left.+\lambda_{i}^{k} \epsilon\left(u_{i}^{k, n+1}\right)\right)
\end{array}
$$

If $x \geq 0, \epsilon(x)=1$, if $x \leq 0, \epsilon(x)=-1$, if $x=0, \epsilon(x)=0$.

Case 3 With a component of a free node (not involved in the contact)

$$
u_{i}^{k, n+1}=\frac{1}{a_{i i}}\left(f_{i}-\sum_{j=1}^{j=i-1} a_{i j} u_{j}^{k, n}-\sum_{j=i+1}^{j=N_{h}} a_{i j} u_{j}^{k, n+1}\right) .
$$

C Convergence test : Yes : $u^{k+1}=u^{k, n+1}$, Go to 3 ; No : Go to B

3 Convergence Test Yes $=$ End; No $=$ Go to 1

At each iteration $k$, the energy present in the discretized convex $\mathcal{K}_{h}$ has to be minimized. This step is performed using the relaxation procedure presented above. Since the functional is strictly convex, the minimization procedure has a unique solution. 
Mixed formulation (see Alart and Curnier (1991); Alart and Lebon (1995))

The idea underlying this method is to write the problem as an equilibrium equation,

$$
\left\{\begin{array}{c}
\text { Find } u \text { and } \lambda \text { such that } \\
F^{\text {int }}(u)+F^{e x t}+\mathcal{F}([u], F)=0, \\
a(F-\mathcal{F}([u], F))=0,
\end{array}\right.
$$

where $a$ is a given coefficient, $F^{e x t}$ are the given external forces, $F^{i n t}$ are internal forces which depend on the constitutive equation and the kinematics, and $\mathcal{F}([u], \lambda)$ is the friction map. In the case of a node in contact with a rigid obstacle (i.e. $[u]=u$ ), in elasto-statics, we have (see Alart and Curnier (1991); Alart and Lebon (1995))

$$
\mathcal{F}(u, F)=\operatorname{proj}_{R_{-}}\left(\tau_{N}\right) n+\operatorname{proj}_{C\left(\operatorname{proj}_{R_{-}}\left(\tau_{N}\right)\right)}\left(\tau_{T}\right),
$$

proj is the projection operator, $C\left(F_{N}\right)$ is the Coulomb's cone and $r$ is a parameter (usually equal to $-r^{-1}$ ). $\tau_{N}$ et $\tau_{T}$ are given by

$$
\tau_{N}=F_{N}+r u_{N}, \tau_{T}=F_{T}+r u_{T} .
$$

The non-linear problem is then written

$$
\mathcal{D}(x)+\mathcal{U}(x)=0
$$

where

$$
\mathcal{D}(x)=\left[\begin{array}{c}
A u+f^{e x t} \\
a F
\end{array}\right] \text { and } \mathcal{U}(x)=\left[\begin{array}{c}
\mathcal{F}(u, F) \\
-a \mathcal{F}(u, F)
\end{array}\right] .
$$

This system is solved using a Generalized Newton method

$$
x^{i+1}=x^{i}-\left(\nabla \mathcal{D}\left(x^{i}\right)+\partial \mathcal{U}\left(x^{i}\right)\right)^{-1}\left(\mathcal{D}\left(x^{i}\right)+\mathcal{U}\left(x^{i}\right)\right)
$$

$\partial \mathcal{U}\left(x^{i}\right)$ is a point in the Jacobian set of matrices. The linearized system (19) is solved by a solver dedicated to non symmetric systems. The algorithm can be summed up as follows:

0 Initialization $x^{0}=\left(u^{0}, \lambda^{0}\right)$ be given

1 Resolution (Non-symmetric linear system)

$$
y^{i+1}=\left(\nabla \mathcal{D}\left(x^{i}\right)+\partial \mathcal{U}\left(x^{i}\right)\right)^{-1}\left(\mathcal{D}\left(x^{i}\right)+\mathcal{U}\left(x^{i}\right)\right)
$$

2 Updating $x^{i+1}=x^{i}-y^{i+1}$

3 Convergence Test Yes $=$ End; No $=$ Go to Step 1 
Concluding comment The numerical problem can be solved using the open computer code LMGC90 (http://www.lmgc.univ-montp2.fr/ ${ }^{\text {dubois- }}$ /LMGC90/). This code is a numerical platform dedicated to the modeling and simulation of dynamic multibody problems. Problems are approached in the general framework of dynamics (see Moreau (1988); Jean (1999)). The discretized equations involved in the problem are written:

$$
\begin{aligned}
M \ddot{q} & =F(q, \dot{q})+P(t)+r, \\
& + \text { interface conditions } \\
& + \text { initial conditions and boundary conditions }
\end{aligned}
$$

where $q$ is a parametrization of the system (degrees of freedom), $M$ is the mass matrix, $F(q, \dot{q})+P(t)$ are the internal and external loading vectors, and $r$ is the vector of contact forces.

The problem of the 105 blocks presented in the previous section can be solved using this technique. The results are presented in figure 10. These results were obtained by J. J. Moreau (Jean and Moreau (1994)). The correspondence observed seems to be perfect.
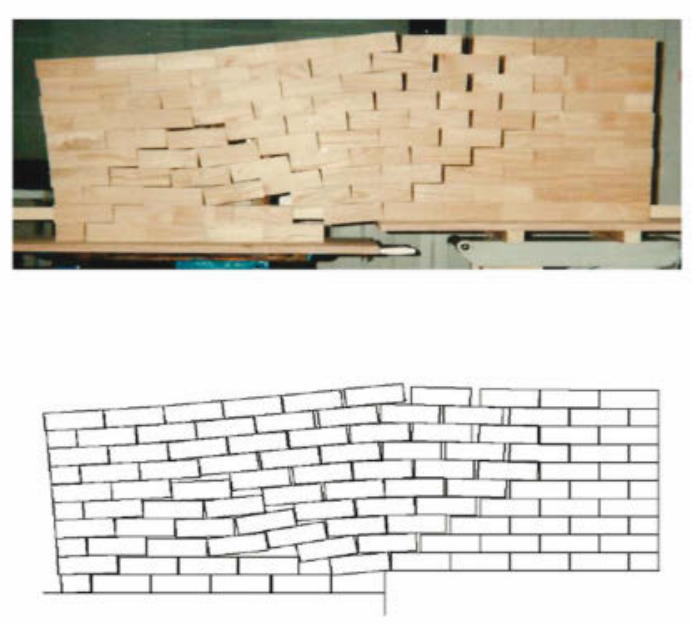

Figure 10. Comparison between experimental and numerical findings on the example of 105 blocks (Jean and Moreau (1994)) 


\section{Masonry blocks with mortar}

\subsection{A phenomenological model taking the adhesion into account (model 2)}

In this section, the RCCM model is presented (Raous et al. (1999); Monerie and Raous (2000)). This adhesion model was used to model mortar/brick interfaces in Fouchal et al. (2009). In this model, the unilateral contact conditions (non penetration between the mortar and the brick, as in the previous section) are combined with dry friction and adhesion between bricks and mortar. The local constitutive equations required for this model are deduced from thermodynamic considerations and based on a material surface hypothesis on the contact zone. The basic idea underlying this model is to introduce a new state variable describing the contact state. This adhesion intensity variable, denoted $b$, was initially introduced by Frémond (1987). This variable gives the relative proportion of the active links between two bodies in contact. This variable is chosen so that:

- $b=1$, total adhesion

- $0<b<1$, partial adhesion

- $b=0$, no adhesion

As in the previous section, we are working in the framework of the contact between two deformable solids, and the same notations are adopted here. $w$ is taken to denote the Dupré energy, and $C_{N}$ (resp. $C_{T}$ ) to denote the initial normal (resp. tangential) stiffness of the interface. In what follows, $p, q$ and $r$ are three given numbers. The constitutive equations of the interface are given by the following equations, based on state laws and complementarity laws:

\section{Unilateral contact with adhesion}

$$
R_{N}-C_{N}\left[u_{N}\right] b^{p} \geq 0 ; \quad\left[u_{N}\right] \geq 0 ; \quad\left(R_{N}-C_{N}\left[u_{N}\right] b^{p}\right)\left[u_{N}\right]=0
$$

\section{Friction with adhesion}

$$
\begin{gathered}
\left\|R_{T}-C_{T}\left[u_{T}\right] b^{q}\right\| \leq \mu\left|R_{N}-C_{N}\left[u_{N}\right] b^{p}\right| \\
\left\|R_{T}-C_{T}\left[u_{T}\right] b^{q}\right\|<\mu \mid R_{N}-C_{N}\left[u_{N}\right] b^{p} \Rightarrow \quad \Rightarrow \quad\left[u_{T}\right]=0 \\
\left\|R_{T}-C_{T}\left[u_{T}\right] b^{q}\right\|=\mu \mid R_{N}-C_{N}\left[u_{N}\right] b^{p} \Rightarrow \quad \exists \lambda \geq 0, \\
{\left[\dot{u_{T}}\right]=\lambda\left(R_{T}-C_{T}\left[u_{T}\right] b^{q}\right)}
\end{gathered}
$$

Evolution of the intensity of adhesion

$$
0=-\left(w-\left(\frac{1}{2} C_{N}\left[u_{T}\right]^{2}+\frac{1}{2} C_{T}\left|\left[u_{T}\right]\right|^{2}\right) b^{r}\right)^{-} \quad \text { if } \quad b \in[0,1[
$$


Note that if there is no adhesion $(b=0)$, this model involves the classical Signorini-Coulomb problem.

A graphic interpretation of the normal part of this RCCM model given in figure 11 (for $(p, q, r)=(2,2,1))$ shows the changes with time in the normal forces depending on the normal displacement jump. The changes in $b$ lead to irreversible effects. If $b$ decreases, the adhesive forces will decrease and eventually disappear. In the case of pure traction $\left(\left[u_{N}\right]>0\right)$, the adhesion resistance $\left(R_{N}=C_{N}\left[u_{N}\right]^{p}\right)$ is activated (elasticity without damage). $b$ decreases when the displacement becomes sufficiently large for the elastic energy to become larger than the adhesion limit $w$.

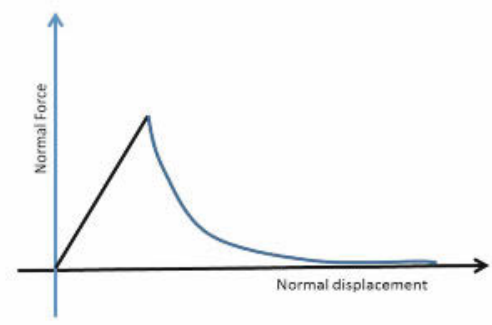

Figure 11. Normalized normal forces versus the normalized normal jump in the displacement

A numerical example (see Fouchal et al. (2009))

Problems are usually approached using $\theta$-methods and the Non-Smooth Contact Dynamics (NSCD) method (Moreau (1988); Jean (1999)). Due to the contact conditions, a fairly small time-step is chosen and the problem is condensed in the local frame associated with the contact nodes. The local problem is solved using a non-linear Gauss-Seidel method.

The interface is governed by the RCCM law presented above. Contact between bodies is defined by contact nodes. Contact nodes are located between two nodes in the mesh of an element in contact at distances of 0.2 and 0.8 , respectively, along each of the segments in contact.

The bodies (bricks and mortar) are modeled using $Q 4$ quadrangular finite elements. The numerical tests are performed with a constant time step equal to $\Delta t=0.5 * 10^{-3} \mathrm{~s}$. The computations require 2000 increments in 
order to reach values resembling the experimental data, and $\theta$ (in the time integration method) is fixed and taken to be equal to 0.55 .

The example presented shows the evolution of a triplet of full bricks and comparisons are made in figures 12 and 13 between two experiments and the numerical results.

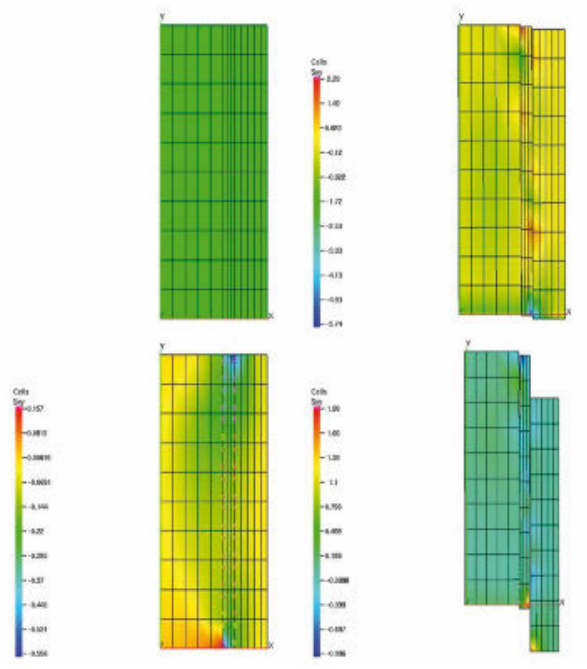

Figure 12. Damage evolution in a triplet of full-bricks

\subsection{Deductive models: linear (and non linear) multi-scale models}

Generalities on asymptotic methods There exist a large class of asymptotic methods, such as matched asymptotic expansions (Eckhaus (1979); Sanchez-Hubert and Sanchez-Palencia (1992)). The general idea is to dilate (blow up) the interphase from the thickness $\eta$ to 1 (see Figure 14). We have two expansions of the displacement $u^{\eta}$, the strain $e\left(u^{\eta}\right)$ and the stress $\sigma^{\eta}$ in the powers of $\eta$, that is, an external one in the adherents and an internal in the joint. We have to connect these two expansions along the interface. In what follows, we study a problem in 2 dimensions in order to simplify the computations. The relations obtained in the internal expansions will be expressed using values that intervene in the external expansions.

a) External expansions The external expansion is a classical expansion 

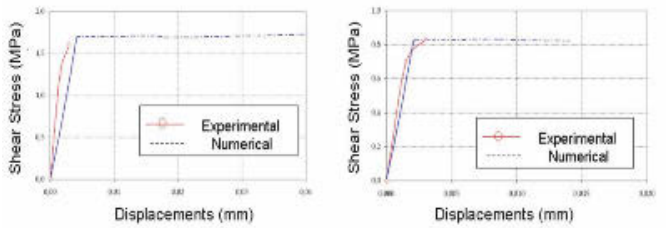

Figure 13. Comparison between experimental and numerical results obtained on full brick triplets

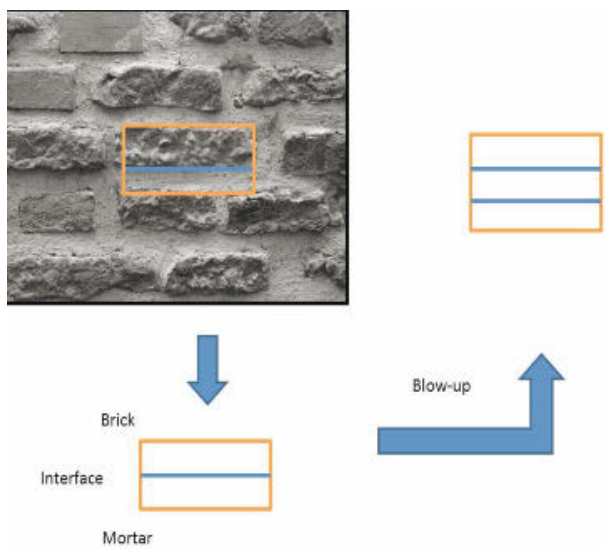

Figure 14. Blow-up process 
in powers of $\eta$

$$
\begin{aligned}
u^{\eta}\left(x_{1}, x_{2}\right) & =u^{0}\left(x_{1}, x_{2}\right)+\eta u^{1}\left(x_{1}, x_{2}\right)+\ldots, \\
e_{i j}\left(u^{\eta}\right)\left(x_{1}, x_{2}\right) & =e_{i j}^{0}+\eta e_{i j}^{1}+\ldots, \\
e_{i j}^{l} & =\frac{1}{2}\left(\frac{\partial u_{i}^{l}}{\partial x_{j}}+\frac{\partial u_{j}^{l}}{\partial x_{i}}\right), \\
\sigma_{i j}^{\eta}\left(x_{1}, x_{2}\right) & =\sigma_{i j}^{0}\left(x_{1}, x_{2}\right)+e \sigma_{i j}^{1}\left(x_{1}, x_{2}\right)+\ldots
\end{aligned}
$$

b) Internal expansions In the internal expansion, we perform a blow-up of the second variable. Let $y_{2}=\frac{x_{2}}{e}$. The internal expansion gives

$$
\begin{aligned}
u^{\eta}\left(x_{1}, x_{2}\right) & =v^{0}\left(x_{1}, y_{2}\right)+\eta v^{1}\left(x_{1}, y_{2}\right)+\ldots, \\
\varepsilon_{i j}\left(u^{\eta}\right)\left(x_{1}, y_{2}\right) & =\eta^{-1} e_{i j}^{-1}+e_{i j}^{0}+e \varepsilon_{i j}^{1}+\ldots, \\
e_{11}^{l} & =\frac{\partial v_{1}^{l}}{\partial x_{1}}, \\
e_{22}^{l} & =\frac{\partial v_{2}^{l+1}}{\partial y_{2}}, \\
e_{12}^{l} & =\frac{1}{2}\left(\frac{\partial v_{2}^{l}}{\partial x_{1}}+\frac{\partial v_{1}^{l+1}}{\partial y_{2}}\right), \\
\sigma_{i j}^{\eta}\left(x_{1}, y_{2}\right) & =\eta^{-1} \tau_{i j}^{-1}\left(x_{1}, y_{2}\right)+\tau_{i j}^{0}\left(x_{1}, y_{2}\right)+\eta \tau_{i j}^{1}\left(x_{1}, y_{2}\right)+\ldots, \\
\sigma_{i j, j}^{\eta} & =\sum_{l=-2}^{\infty} \eta^{l}\left(\frac{\partial \tau_{i 1}^{l}}{\partial x_{1}}+\frac{\partial \tau_{i 2}^{l+1}}{\partial y_{2}}\right) .
\end{aligned}
$$

We use the convention

$$
v^{l}=0, l<0, \tau^{l}=0, l<-1 .
$$

c) Continuity conditions The third step in the method consists in the connecting of the two expansions. If the interface between the mortar and the brick is perfect, we have continuity of the displacement and of the stress tensor along this interface. This gives:

$$
\begin{aligned}
(i) v^{0}\left(x_{1}, \pm 1 / 2\right) & =u^{0}\left(x_{1}, \pm 1 / 2\right) \\
(i i) \tau^{-1}\left(x_{1}, \pm \pm 1 / 2\right) & =0 \\
(\text { ii }) \tau^{0}\left(x_{1}, \pm 1 / 2\right) . e_{2} & =\sigma^{0}\left(x_{1}, \pm 1 / 2\right) . e_{2} .
\end{aligned}
$$

A linear multi-scale model (model 3) The equilibrium equation becomes:

$$
\left(\eta^{-1} \tau_{i j}^{-1}+\tau_{i j}^{0}+\eta \tau_{i j}^{1}+\ldots\right)_{, j}=0
$$

We obtain

$$
\begin{array}{cc}
\tau_{i j}^{-1} & =0 \\
\tau_{i 2,2}^{0} & =0
\end{array}
$$

that is 


$$
\left[\tau_{i 2}^{0} \cdot e_{2}\right]=0
$$

The interphase is assumed to be linearly elastic, and the above theory is applied to this kind of material. We have

$$
\eta^{-1} \tau_{i j}^{-1}+\tau_{i j}^{0}+\eta \tau_{i j}^{1}+\ldots=C_{i j k l}\left(\eta^{-1} e_{k l}^{-1}+e_{k l}^{0}+\eta e_{k l}^{1}+\right)
$$

where $C$ denotes the stiffness tensor.

We obtain:

$$
\begin{aligned}
& \tau_{12}^{0}=C_{1212} v_{1,2}^{0} \\
& \tau_{22}^{0}=C_{2222} v_{2,2}^{0}
\end{aligned}
$$

that is

$$
\begin{aligned}
& \tau_{12}^{0}=C_{1212}\left[v_{1}^{0}\right] \\
& \tau_{22}^{0}=C_{2222}\left[v_{2}^{0}\right]
\end{aligned}
$$

Using the continuity conditions, we obtain

$$
\begin{aligned}
\sigma_{i j}^{0} & =0 \text { in the brick and the mortar } \\
\sigma^{0} . e_{2} & =\widehat{C}\left[u^{0}\right] \text { along the interface }
\end{aligned}
$$

where $\widehat{C}$ is a matrix consisting of $C_{1212}$ and $C_{2222}$. Note that this technique was used in Rekik and Lebon (2010) and Rekik and Lebon (2012).

An example of a non linear multi-scale model including microcracks (model 4) (see Pelissou and Lebon (2009))

General considerations and notations

The model described in this section is an extension of the bulk model introduced in Gambarotta and Lagomarsino (1997), which takes the damage to the mortar joint into account. The interface modeling procedure (figure 15) consists of three steps:

- Let us take a macroscopic bulk model for quasi-fragile materials;

- The structure is assumed to consist of three phases: material 1 (brick, for example), material 2 (mortar, for example) and a thin interphase between the two materials, consisting of the material described in the first step;

- Since the interphase is thin, an interface model is developed by performing an asymptotic analysis, as described above (Geymonat and Krasucki (1997); Lebon and Ronel-Idrissi (2004)) (the thickness of the interface tends to zero). 


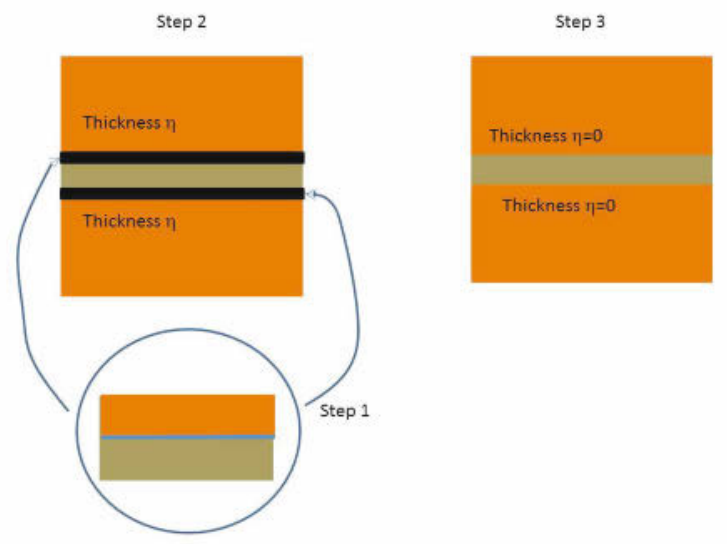

Figure 15. Interface modeling in three steps

The model obtained memorizes some of the geometrical and mechanical characteristics of the interphase, such as the thickness, elastic coefficients, normal and tangential stress, damage variable, etc.

For the sake of simplicity, the structure is taken to occupy an open bounded set $\Omega$ of $\Re^{2}$ with a smooth boundary $\partial \Omega$. The two dimensional space is referred to the orthonormal frame $\left(O, x_{1}, x_{2}\right)$.

First step: The bulk model

In this section, the bulk model introduced by Gambarotta and Lagomarsino (1997) is briefly described. In this model dedicated to masonry structures, the masonry is regarded as a "material" showing nonlinear damage behavior. The macroscopic behavior is accounted for by applying averaging process based on microscopic considerations. In the line with the classical procedure, the strain tensor is decomposed in its linear and nonlinear (anelastic) parts:

$$
e=e^{e l}+e^{a n}
$$

where

$$
\sigma=C e^{e l} \text { and } e^{a n}=S_{\sigma} \sigma
$$

At the local level (in an elementary volume, see figure 16), the stress vector is decomposed into its normal $\sigma_{N}$ and tangential $\sigma_{T}$ parts, and the normal and tangential components of $\boldsymbol{e}^{\boldsymbol{a n}}$ are expressed by (37) :

$$
\left\{\begin{array}{l}
e_{N}^{a n}=h \alpha H\left(\sigma_{N}\right) \sigma_{N} \\
e_{T}^{a n}=k \alpha\left(\sigma_{T}-f\right)
\end{array}\right.
$$


where $f=\sigma_{T}$ if $\left.\sigma_{T} \in I=\right]-\mu \sigma_{N},+\mu \sigma_{N}$ [and $f= \pm \mu \sigma_{N}$ if $\sigma_{T} \notin I$ (figure 17), $H$ is the Heaviside function of the unilateral response of the joint and $\alpha$ is the damage variable. $h$ and $k$ are positive coefficients standing for the opening and sliding compliances of the mortar joint and $\mu$ is the internal friction coefficient.

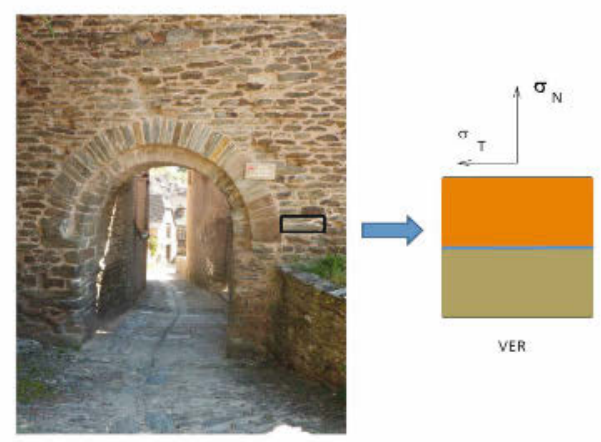

Figure 16. Normal and tangential stress vector components

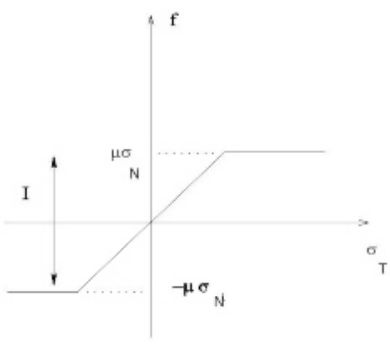

Figure 17. Relation between $f$ and $\sigma_{T}$ (friction threshold)

We can write

$$
e_{t}^{a n}=k \alpha \chi_{I}\left(\sigma_{T}\right)\left(\sigma_{T} \pm \mu \sigma_{N}\right)
$$




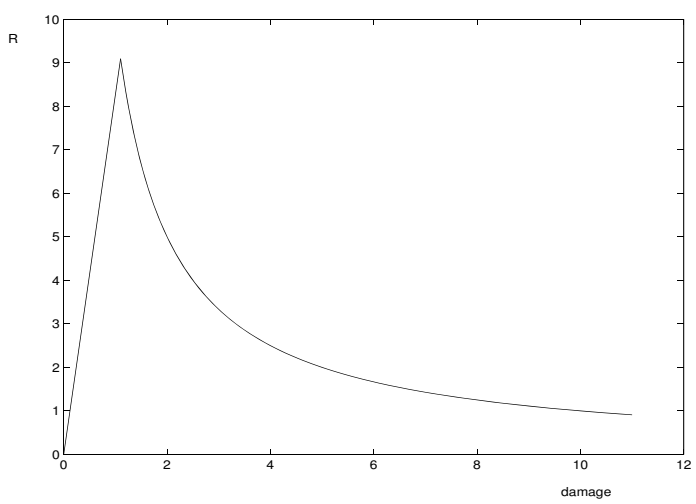

Figure 18. Evolution of $R(\alpha)$

where $\chi_{I}$ is the characteristic function of the tangential damage undergone by the set $I$, such that:

- If $\sigma_{T} \in I, \chi_{I}(\tau)=1$, there is tangential damage.

- If $\sigma_{T} \notin I, \chi_{I}\left(\sigma_{T}\right)=0$, there is no tangential damage.

Therefore $S_{\sigma}$ is given locally in matrix form by:

$$
S_{\sigma}=\left(\begin{array}{cc}
h \alpha H\left(\sigma_{N}\right) & 0 \\
\pm k \alpha \chi_{I}\left(\sigma_{T}\right) \mu & k \alpha \chi_{I}\left(\sigma_{T}\right)
\end{array}\right)
$$

The damage is governed by a yield condition $\Phi(\alpha) \leq 0$, where

$$
\begin{aligned}
\Phi(\alpha) & =Y-R(\alpha) \\
Y & =\frac{1}{2} h H\left(\sigma_{N}\right) \sigma_{N}^{2}+\frac{1}{2} k \chi_{I}\left(\sigma_{T}\right) \sigma_{T}^{2}
\end{aligned}
$$

where $R$ is the toughness of the material defined by (see figure 18)

$$
\begin{aligned}
& R(\alpha)=R_{0} \alpha, \quad \text { if } 0 \leq \alpha \leq 1 \\
& R(\alpha)=R_{0} / \alpha, \quad \text { if } \alpha \geq 1
\end{aligned}
$$

Second step: The interphase

In this section, the structure is taken to consist of two materials separated by a thin interphase which is a "mixture" of the other two materials. The interphase is assumed to be parallel to the $x_{1}$-axis and the thickness, which is constant, is denoted by $\eta$. In what follows, the constitutive equations are those given in the previous section, i.e., the interface consists of a 
quasi-brittle material. The elastic part is assumed to be isotropic, and in view of the external normal vector $x_{2}$, the constitutive equation is

$$
\sigma=\left(I d+C S_{\sigma}\right)^{-1} C e=C_{\sigma} \varepsilon
$$

Upon introducing the Lamé's coefficients, $\lambda$ and $G$, we can write:

$$
C_{\sigma}=\left(\begin{array}{ccc}
\lambda+2 G-\frac{\lambda^{2} h \alpha H}{1+(\lambda+2 G) h \alpha H} & \lambda-\frac{\lambda(\lambda+2 G) h \alpha H}{1+(\lambda+2 G) h \alpha H} & 0 \\
\frac{\lambda}{1+(\lambda+2 G) h \alpha H} & \frac{\lambda+2 G}{1+(\lambda+2 G) h \alpha H} & 0 \\
-\frac{ \pm \mu \lambda G k \alpha \chi_{I}}{(1+(\lambda+2 G) h \alpha H)\left(1+G k \alpha \chi_{I}\right)} & -\frac{ \pm \mu(\lambda+2 G) G k \alpha \chi_{I}}{(1+(\lambda+2 G) h \alpha H)\left(1+G k \alpha \chi_{I}\right)} & \frac{G}{1+G k \alpha \chi_{I}}
\end{array}\right)
$$

\section{Interface behavior}

Using this asymptotical approach (see the previous section or Lebon and Ronel-Idrissi (2004) in another context), and substituting the asymptotic expansions into the constitutive equations and the equilibrium equations, we obtain expressions linking the stress vector to the jump in the displacement denoted $[u]$. This gives:

$$
\begin{aligned}
\tau_{22}^{0} & =\lim _{e \rightarrow 0} \frac{\lambda+2 G}{1+(\lambda+2 G) h \alpha H} \frac{\partial v_{2}^{0}}{\partial y_{2}} \\
\tau_{12}^{0} & =\lim _{e \rightarrow 0} \frac{ \pm \mu(\lambda+2 G) G k \alpha \chi_{I}}{(1+(\lambda+2 G) h \alpha H)\left(1+G k \alpha \chi_{I}\right)} \frac{\partial v_{2}^{0}}{\partial y_{2}}+\lim _{e \rightarrow 0} \frac{G}{1+G k \alpha \chi_{I}} \frac{\partial v_{1}^{0}}{\partial y_{2}}
\end{aligned}
$$

By integration, the A-GL (Asymptotic Gambarotta-Lagomarsino) model is therefore given by the following system (in the terms of the normal and tangential components):

$$
\left\{\begin{aligned}
\sigma_{N} & =\frac{C_{N}}{1+C_{N} \bar{h} \alpha H\left(\sigma_{N}\right)}\left[u_{N}\right] \\
\sigma_{T} & =\frac{ \pm C_{N} C_{T} \mu \bar{k} \alpha \chi_{I}}{\left(1+C_{N} \bar{h} \alpha H\left(\sigma_{N}\right)\right)\left(1+C_{T} \bar{k} \alpha \chi_{I}\right)}\left[u_{N}\right]+\frac{C_{T}}{1+C_{T} \bar{k} \alpha \chi_{I}}\left[u_{T}\right]
\end{aligned}\right.
$$

where

$$
\begin{aligned}
C_{N} & =(\lambda+2 G) / e \\
C_{T} & =G / e \\
\bar{h} & =h e \\
\bar{k} & =k e
\end{aligned}
$$

The resulting matrix is not diagonal, contrary to the classical case: a non symmetric coupling term occurs between the normal compliance term $C_{N}$ and the tangential compliance term $C_{T}$. It can be noted that the configuration of traction when $f=0$ is combined in this general expression with 
that of the compression when $f=\sigma_{T}$ and when the friction threshold is reached at $f= \pm \mu \sigma_{N}$.

The damage is governed by a yield condition $\bar{\Phi}(\alpha)=\bar{Y}-\bar{R}(\alpha) \leq 0$, where

$$
\left\{\begin{array}{c}
\bar{Y}=\frac{1}{2} \bar{h} H\left(\sigma_{N}\right) \sigma_{N}^{2}+\frac{1}{2} \bar{k} \chi_{I}\left(\sigma_{T}\right)\left(\sigma_{T}- \pm \mu \sigma_{N}\right)^{2} \\
\bar{R}(\alpha)=\lim _{e \rightarrow 0} R e= \begin{cases}\bar{R}_{0} \alpha & \text { if } 0<\alpha<1 \\
\frac{\bar{R}_{0}}{\alpha} & \text { if } \alpha>1\end{cases}
\end{array}\right.
$$

A numerical example: shear test on a simple brick Let us perform an academic shear test on a single rectangular piece. The piece is bonded at the bottom and subjected to horizontal forces on the left. Details of this test are presented in figure 19. This horizontal force which is equal to $20 k N$, is applied progressively. The load is applied on the right and left sides. The (academic) coefficients are $C_{N}=C_{T}=500 \mathrm{kNcm}^{-3}, \mu=0.3, \bar{h}=\bar{k}=$ $0.04 \mathrm{kN}^{-1} \mathrm{~cm}^{3}, \bar{R}_{0}=10 \mathrm{kNcm} \mathrm{cm}^{-1}$.
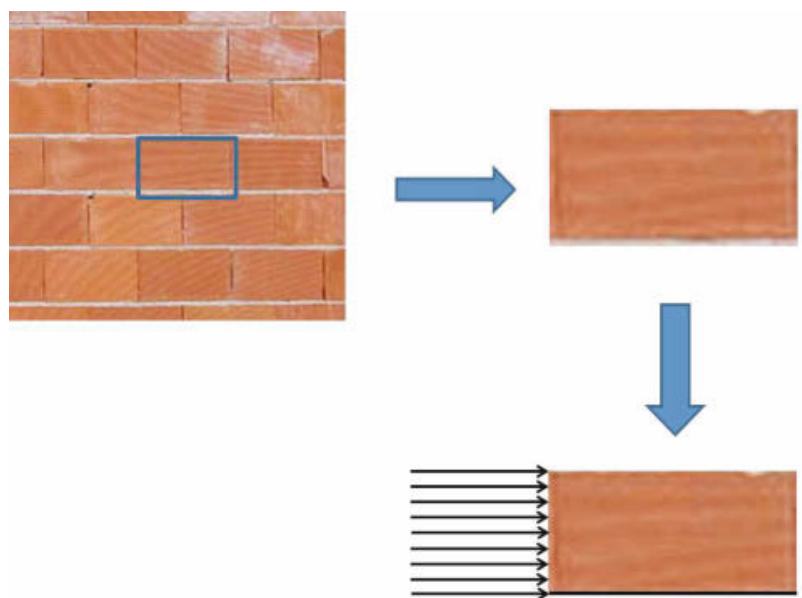

Figure 19. Shear test

Due to the geometry and the loading conditions, the behavior of the contact zone is very complex. Figure 20 shows the evolution of the damage along the contact zone, which is strongly non linear. In particular, 
the damage increases strongly in an intermediate zone, which is subjected to both shear and traction forces. Note that the tangential displacement shows a linear pattern of evolution in the first phase and a non linear pattern corresponding to the failure, in the second phase, as was to be expected.

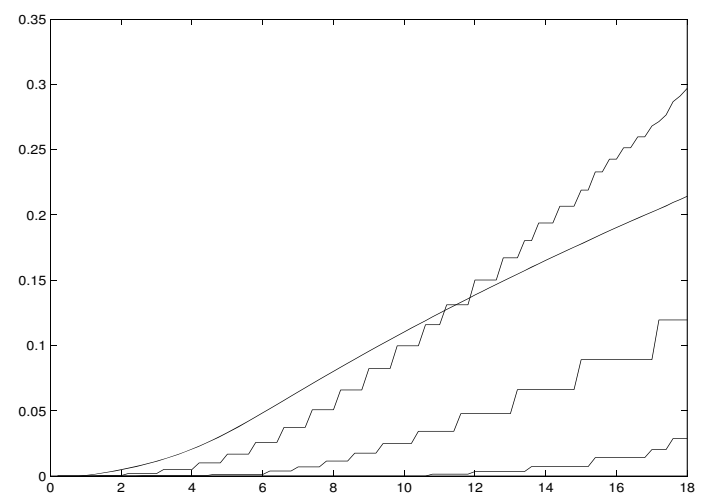

Figure 20. Damage evolution for four typical elements (shear test).

\section{Conclusion}

In this chapter, four of the many existing interface models are presented. It is quite difficult to solve large problems using this approach. In Rekik and Lebon (2010, 2012), we established that it can be possible to solve larger problems (small walls), however. It will obviously be necessary in future studies to review all the existing models (a simple look at Science Direct ${ }^{T M}$ with the keywords "masonry" and "interface" gives more than 2500 papers), in order to choose the most suitable one and use efficient solvers (DDM, MG, etc.) before it will be possible to solve real physical problems.

\section{Bibliography}

P. Alart and A. Curnier. A mixed formulation for frictional contact problems prone to Newton like solution methods. Computer Methods in Applied Mechanics and Engineering, 92:353-375, 1991.

P. Alart and F. Lebon. Solution of frictional contact problems using ILU and coarse/fine preconditioners. Computational Mechanics, 16:98-105, 1995 . 
E. Bonetti and M. Frémond. Analytical results on a model for damaging in domains and interfaces. ESAIM-Control Optimisation and calculus of Variations, 17:955-974, 2011.

P. Chabrand, F. Dubois, and M. Raous. Various numerical methods for solving unilateral contact problems with friction. Mathematical Computer and Modeling, 28(4-8):97-108, 1998.

M. Cocu. Existence of solutions of Signorini problems with friction. International Journal of Engineering Science, 22:567-575, 1984.

M. Cocu, E. Pratt, and M. Raous. Formulation and approximation of quasistatic frictional contact. Comptes Rendus Académie des Sciences, Paris, Série I, 320:1413-1420, 1995.

M. Como. Statica delle costruzioni storiche in muratura. Aracne, 2010.

G. Duvaut and J. L Lions. Inequalities in mechanics and physics. Springer Verlag, 1976.

W. Eckhaus. Asymptotic analysis of singular perturbations. North-Holland, 1979.

J. Fortin, M. Hjiaj, and G. De Saxcé. An improved discrete element method based on a variational formulation of the frictional contact law. Computers and Geotechnics, 29:609-640, 2002.

F. Fouchal. Contribution à la modélisation numérique des interfaces dans les structures maçonnées. PhD thesis, Université de Reims Champagne Ardennes, 2006.

F. Fouchal, F. Lebon, and I. Titeux. Contribution to the modeling of interfaces in masonry construction. Construction and Building Materials, 23:2428-2441, 2009.

F. Freddi and M. Frémond. Damage in domains and interfaces: A coupled predictive theory. Journal of Mechanics of Materials and Structures, 1: 1205-1233, 2006.

M. Frémond. Adhesion of solids. Journal de Mécanique Théorique et Appliquée, 6:383-407, 1987.

A. Gabor. Contribution à la caractérisation et à la modélisation des maçonneries non renforcées et renforcées par materiaux composites. $\mathrm{PhD}$ thesis, Université Claude-Bernard Lyon I, 2002.

A. Gabor, A. Bennani, E. Jacquelin, and F. Lebon. Modelling approaches of the in-plane shear behaviour of unreinforced and frp strengthened masonry panels. Composites Structures, 74:277-288, 2006.

L. Gambarotta and S. Lagomarsino. Damage models for the seismic response of brick masonry walls. part i : The continuum model and its application. Earthquake Engineering and Structural Dynamics, 26:441-462, 1997.

G. Geymonat and F. Krasucki. Analyse asymptotique du comportement en flexion de deux plaques collées. Compte Rendu Académie des Sciences Série I, 325:307-314, 1997. 
P. Hild. An example of nonuniqueness for the continuous static unilateral contact model with coulomb friction. Comptes Rendus Académie des Sciences, Paris, Série I, 337:685-688, 2003.

M. Jean. The non-smooth contact dynamics method. Computer Methods in Applied Mechanics and Engineering, 177:235-257, 1999.

M. Jean and J. J. Moreau. Monuments under seismic action. Technical report, Commission of the European Communities, November 1994. Environment Programme, Contract n EV5V CT93 0300.

A. Klarbring. Derivation of the adhesively bonded joints by the asymptotic expansion method. International Journal of Engineering Science, 29: 493-512, 1991.

F. Lebon. Two-grid method for regularized frictional elastostatics problems. Engineering Computations, 12:657-664, 1995.

F. Lebon. Contact problems with friction: Models and simulations. Simulation, Modelling, Practice and Theory, 11:449-464, 2003.

F. Lebon and M. Raous. Friction modelling of a bolted junction under internal pressure loading. Computers and Structures, 43:925-933, 1992.

F. Lebon and R. Rizzoni. Asymptotic study of soft thin layer: the non convex case. Mechanics of Advanced Materials and Structures, 15:12-20, 2008.

F. Lebon and S. Ronel. First order numerical analysis of linear thin layers. ASME Journal of Applied Mechanics, 74:824-828, 2007.

F. Lebon and S. Ronel-Idrissi. Asymptotical analysis of Mohr-Coulomb and Drucker-Prager soft thin layers. Steel and Composite Structures, 4: 133-147, 2004.

F. Lebon, A. Ould-Khaoua, and C. Licht. Numerical study of soft adhesively bonded joints in finite elasticity. Computational Mechanics, 21:134-140, 1997.

F. Lebon, R. Rizzoni, and S. Ronel. Analysis of non-linear soft thin interfaces. Computers and Structures, 82:1929-1938, 2004.

C. Licht and G. Michaille. A modeling of elastic adhesive bonded joints. Advances in Mathematical Sciences and Applications, 7:711-740, 1997.

M. Lucchesi, C. Padovani, G. Pasquinelli, and N. Zani. Masonry constructions: mechanical models and numerical applications. Springer, 2008.

Y. Monerie and M. Raous. A model coupling adhesion to friction for the intercation between a crack and a fiber/matrix interface. $Z A M M, 80$ : 205-209, 2000.

J. J. Moreau. Unilateral contact and dry friction in finite freedom dynamics, volume 302 of CISM - Courses and Lectures, pages 1-82. Springer, 1988.

C. Pelissou and F. Lebon. Asymptotic modeling of quasi-brittle interfaces. Computers and Structures, 87:1216-1223, 2009. 
N. Point and E. Sacco. A delamination model for laminated composite. International Journal of Solids and Structures, 33:483-509, 1996.

M. Raous. Interface models coupling adhesion and friction. Comptes Rendus Mécanique, 339:491-501, 2011.

M. Raous, P. Chabrand, and F. Lebon. Numerical methods for solving unilateral contact problem with friction. Journal of Theoretical and Applied Mechanics, 7:111-128, 1988.

M. Raous, L. Cangémi, and M. Cocou. A consistent model coupling adhesion, friction and unilateral contact. Compututer Methods in Applied Mechanics and Engineering, 177:383-399, 1999.

A. Rekik and F. Lebon. Identification of the representative crack length evolution in a multi-level interface model for quasi-brittle masonry. International Journal of Solids and Structures, 47:3011-3021, 2010.

A. Rekik and F. Lebon. Homogenization methods for interface modeling in damaged masonry. Advances in Engineering Software, 46:35-42, 2012.

J. Sanchez-Hubert and E. Sanchez-Palencia. Introduction aux méthodes asymptotiques et à l'homogénisation. Masson, 1992.

P. Wriggers. Computational Contact Mechanics. Springer, 2006. 